\title{
Study on Parallel Computing
}

\author{
Guoliang Chen \\ University of Science and Technology of China
}

\begin{abstract}
In this talk, we present a general survey on parallel computing. The main contents include parallel computer system which is the hardware platform of parallel computing, parallel algorithm which is the theoretical base of parallel computing, parallel programming which is the software support of parallel programming, parallel application which is the development impetus of parallel computing. Specially, we also introduce some enabling technologies of parallel application. We argue that the parallel computing research should form an integrated methodology of "architecture-algorithm-programming-application". Only in this way, parallel computing research becomes continuous development and more realistic.
\end{abstract}

